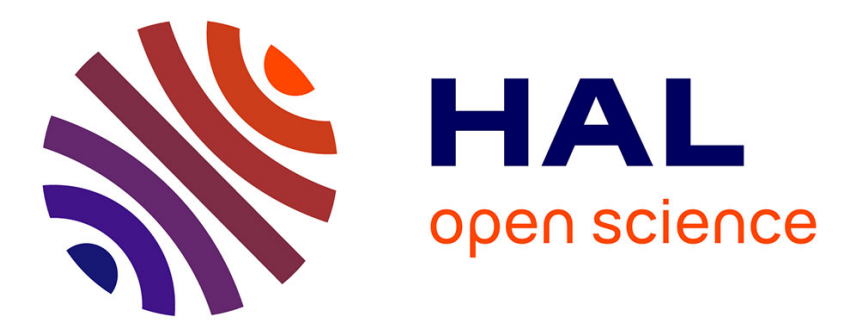

\title{
A modular wearable finger interface for cutaneous and kinesthetic interaction: control and evaluation
}

\author{
Francesco Chinello, Monica Malvezzi, Domenico Prattichizzo, Claudio
}

Pacchierotti

\section{- To cite this version:}

Francesco Chinello, Monica Malvezzi, Domenico Prattichizzo, Claudio Pacchierotti. A modular wearable finger interface for cutaneous and kinesthetic interaction: control and evaluation. IEEE Transactions on Industrial Electronics, 2020, 67 (1), pp.706-716. 10.1109/TIE.2019.2899551 • hal-02021347

\section{HAL Id: hal-02021347 \\ https://hal.inria.fr/hal-02021347}

Submitted on 15 Feb 2019

HAL is a multi-disciplinary open access archive for the deposit and dissemination of scientific research documents, whether they are published or not. The documents may come from teaching and research institutions in France or abroad, or from public or private research centers.
L'archive ouverte pluridisciplinaire HAL, est destinée au dépôt et à la diffusion de documents scientifiques de niveau recherche, publiés ou non, émanant des établissements d'enseignement et de recherche français ou étrangers, des laboratoires publics ou privés. 


\title{
A modular wearable finger interface for cutaneous and kinesthetic interaction: control and evaluation
}

\author{
Francesco Chinello, Member, IEEE, Monica Malvezzi, Member, IEEE, \\ Domenico Prattichizzo, Fellow, IEEE, and Claudio Pacchierotti, Member, IEEE
}

\begin{abstract}
We present a novel modular wearable interface for haptic interaction and robotic teleoperation. It is composed of a 3-DoF fingertip cutaneous device and a 1DoF finger kinesthetic exoskeleton, which can be either used together as a single device or separately as two different devices. The 3-DoF fingertip device is composed of a static body and a mobile platform. The mobile platform is capable of making and breaking contact with the finger pulp and re-angle to replicate contacts with arbitrarily oriented surfaces. The 1-DoF finger exoskeleton provides kinesthetic force to the proximal and distal interphalangeal finger articulations using one servo motor grounded on the proximal phalanx. This paper presents the wearable device as well as three different position, force, and compliance control schemes, together with their evaluations. We also present three human subjects experiments, enrolling a total of $\mathbf{4 0}$ different participants: the first experiment considered a curvature discrimination task, the second one a robotassisted palpation task, and the third one an immersive experience in Virtual Reality. Results showed that providing cutaneous and kinesthetic feedback through our device significantly improved the performance of all the considered tasks. Moreover, although cutaneous-only feedback showed promising performance, adding kinesthetic feedback improved most metrics. Finally, subjects ranked our device as highly wearable, comfortable, and effective.
\end{abstract}

Index Terms-Haptic interfaces, Exoskeletons

\section{INTRODUCTION}

$\mathbf{H}$ APTIC sensations provided by commercially-available wearable devices are often limited to vibrations, reducing the possibility of rendering complex contact interactions. Toward more realistic touch sensations, researchers started to study how to provide other types of cutaneous stimuli in a wearable and unobtrusive way [1]. One of the first wearables able to provide rich cutaneous sensations has been presented by Minamizawa et al. [2]. Two motors, placed on the nail side

Manuscript received August 27, 2018; revised December 4, 2018; accepted January 31,2019 . This research has received funding from Rennes Métropole (2017 AIS) and from the CNRS (2018 PEPS JCJC).

F. Chinello is with the Dept. Business Development and Technology, Extended Reality and Robotics Lab. $\left(\mathrm{XR}^{2}\right)$, Aarhus Univ., Herning, Denmark. (e-mail: chinello@btech.au.dk).

M. Malvezzi and D. Prattichizzo are with the Dept. Information Eng. and Mathematics, Univ. Siena, Siena, Italy, and with the Dept. Adv. Robotics, Istituto Italiano di Tecnologia, Genova, Italy. (e-mail: \{malvezzi, prattichizzo\}@diism.unisi.it).

C. Pacchierotti is with the CNRS, Univ Rennes, Inria, IRISA, Rennes, France. (e-mail: claudio.pacchierotti@irisa.fr).
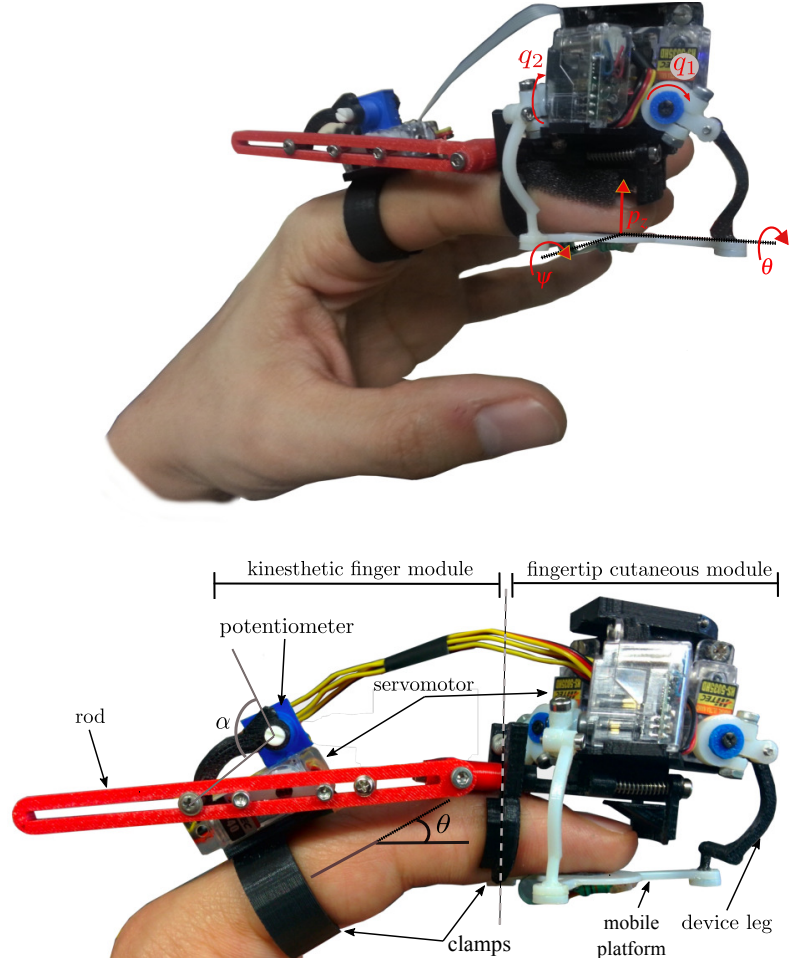

Fig. 1. The proposed wearable finger device. It is composed of a 3-DoF fingertip cutaneous module and a 1-DoF kinesthetic finger module. The 3-DoF fingertip device comprises two platforms: one on the back of the finger (black), supporting three small servo motors, and the other one in contact with the volar surface of the fingertip (white). The 1-DoF finger module is grounded on the proximal phalanx and it provides kinesthetic force to the PIP and DIP joints using one servo motor.

of the fingertip, move a belt in contact with the user's finger pulp. The belt applies a normal force to the user's fingertip when the motors rotate in opposite directions, while it applies a shear force when the motors rotate in the same direction. The device's motor inputs are calculated by implementing a simple proportional law between belt displacement and target stimuli. More recently, Prattichizzo et al. [3] presented a similar cabledriven 3-degrees-of-freedom (3-DoF) cutaneous device for the fingertip. A static platform, housing three DC motors, is located on the nail, while a mobile platform, acting as the end-effector, is placed in contact with the finger pulp. The mobile platform can press into the user's fingertip and re-angle to simulate contacts with slanted surfaces. This device has 
been used for applications in robotic teleoperation and virtual reality, mostly employing position-based control approaches.

Although this type of cutaneous devices have been successfully used in various scenarios [4], [5], their end-effectors always contact the finger skin. As a result, these devices are not capable of conveying the sensation of making and breaking contact with virtual and remote surfaces, which is known to be important for haptic interaction [6]. To overcome this limitation, Kuchenbecker et al. [7] presented a passive contact location display to be attached to a grounded haptic interface. The kinesthetic feedback provided by the grounded interface bends the internal springs of the display and brings a shell in contact with the user's finger, providing the sensation of making and breaking contact with the rendered surface. Frisoli et al. [8] achieved a similar effect by creating a fingermounted thimble that moves a 5-DoF flat contact plate around the fingertip. More recently, Girard et at. [9] developed a 2DoF wearable haptic device able to render shear forces at the fingertip. It is composed of a parallelogram structure actuated by two DC motors which move a peg responsible for the shear feedback. A recent review on wearable haptic interfaces can be found in [1]

To provide well-rounded sensations, researchers have also worked on designing wearable interfaces able to provide both kinesthetic and cutaneous stimuli. For example, Cempini et al. [10] developed an underactuated fingertip exoskeleton with a custom self-alignment mechanism to absorb human/robot joint axes misplacement. It has been designed for close human-robot interaction applications and it is driven using a hierarchical two-layer position controller. Sarac et al. [11] recently presented an underactuated hand exoskeleton able to adapt its shape and size to objects during grasping. It has been designed for applications in virtual and augmented reality. A review of the literature on lightweight exoskeletons for the hand can be found in [1], [12]. Indeed, providing both cutaneous and kinesthetic feedback has often showed better performance than providing either cutaneous or kinesthetic feedback alone. For example, when kinesthetic feedback was enriched with cutaneous cues, Frisoli et al. [8] found a significantly lower threshold for curvature discrimination $\left(1.51 \pm 0.2 \mathrm{~m}^{-1}\right.$ vs. $\left.2.62 \pm 0.61 \mathrm{~m}^{-1}\right)$ for stimuli constituted of spheres with curvatures ranging in the interval 4-6 $\mathrm{m}^{-1}$. Pacchierotti et al. [13] showed that providing both cutaneous and kinesthetic feedback improved the performance of a teleoperated pick and place task with respect to conveying, separately, either kinesthetic or cutaneous feedback. Similarly, Meli et al. [14] found that providing cutaneous stimuli alone performed worse than providing cutaneous and kinesthetic stimuli in a hole-in-peg task. More recently, Quek et al. [15] used a teleoperation system to perform two manipulation tasks (peg transfer and tube connection) using kinesthetic feedback, skin deformation feedback, and the combination of both. The combined kinesthetic and skin deformation feedback achieved better performance and higher participant ratings compared to kinesthetic or skin deformation feedback alone.

This paper presents the control and evaluation of a novel modular interface for haptic interaction, shown in Fig. 1. The device is composed of a 3-DoF fingertip cutaneous device and a 1-DoF finger kinesthetic exoskeleton, which can be either used together as a single device or separately as two different devices. The 3-DoF fingertip module is composed of a static upper body and a mobile platform: the static body is located on the nail, supporting three small servo motors, and the mobile platform is placed in front of the finger pulp. The two parts are connected by three articulated legs, according to a Revolute-Revolute-Spherical (RRS) kinematic chain. The legs are actuated by the servo motors and can move the platform away and toward the user's finger skin as well as rotate it to mimic contacts with arbitrarily oriented surfaces. With respect to the cable-driven fingertip devices presented in [3], [5], our 3-DoF fingertip module solves the indeterminacy due to the underactuation of the platform. Moreover, it is one of the most compact and lightweight device ever presented [1]. The 3DoF fingertip module alone weighs $24 \mathrm{~g}$ for $35 \times 50 \times 43 \mathrm{~mm}$ dimensions, and its design is inspired by [16].

The finger exoskeleton is a 3-DoF planar mechanism: two DoF allow the adaptability to different finger sizes, while the third one provides the actuation. Once the exoskeleton is worn, a static part is fixed on the proximal phalanx, while a mobile part is fixed close to the Distal-Interphalangeal (DIP) joint axis. In this way, the finger kinematic structure, composed of the proximal and intermediate phalanges connected through the Proximal-Interphalangeal (PIP) joint, constraints the exoskeleton kinematics, reducing the overall system mobility to 1 DoF. Consequently, only one motor is needed to actuate the finger/exoskeleton system and provide kinesthetic stimuli to the PIP/DIP articulation. With respect to similar interfaces [10], [11], [17], the proposed 1-DoF finger exoskeleton is extremely compact and lightweight: it weighs only $18 \mathrm{~g}$ for $117 \times 30 \times 42 \mathrm{~mm}$ dimensions. The complete kinesthetic and cutaneous device weighs $42 \mathrm{~g}$ for $117 \times 50 \times 43 \mathrm{~mm}$ dimensions. Moreover, it can be easily adjusted to fingers of different sizes. Finally, to the best of our knowledge, it is the first time two wearable devices are designed in such a modular way. A video showing the device can be found at https://youtu.be/N9_jFOafkKk

\section{CONTROL}

According to the type of interaction to be rendered, our device can be controlled in different ways. We present here four control schemes for our two modules, along with their evaluation. These control schemes will be then used in the experiments of Secs. III and IV.

\section{A. Cutaneous device position control}

The commanded tactile sensations can be provided to the user's fingertip by controlling the position and orientation of the 3-DoF platform. In the underactuated wearable devices of [3], [18], where the platform is controlled by three cables, position and force controls are coupled. In both schemes, the estimation of the platform displacement is approximated assuming a linear contact stiffness model. However, this solution presents several issues. The most evident is that the controller heavily relies on several parameters that cannot be measured or properly estimated, such as the size of the fingertip, or the age 


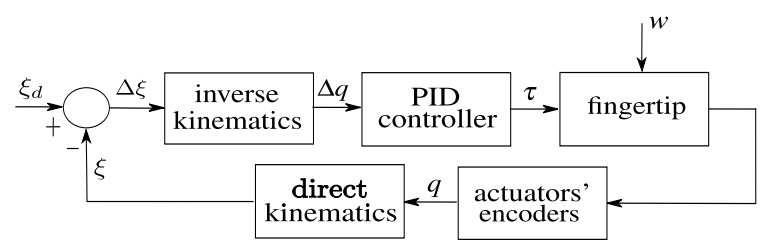

(a) Coupled position control scheme.

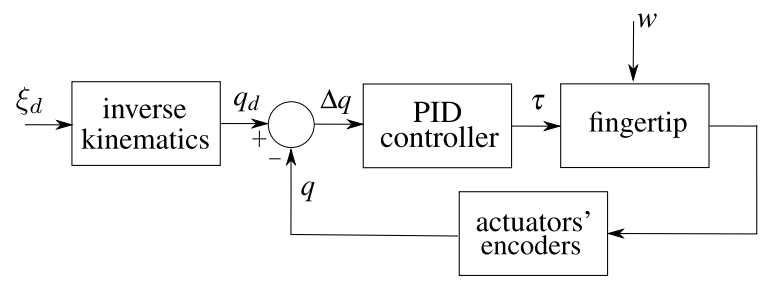

(b) Decoupled position control scheme.

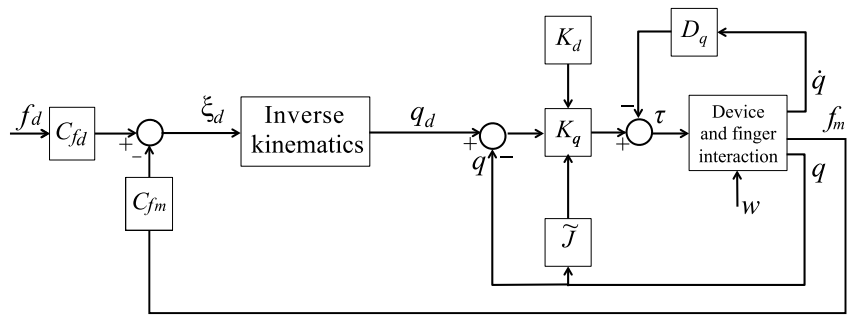

(c) Force control scheme.

Fig. 2. 3-DoF cutaneous device. Position and force control schemes.

and sex of the user. The 3 DoF device presented in this paper, which is not underactuated, overcomes this indeterminacy. Its position and force control approaches can be in fact decoupled, and they do not require a model of the fingertip compliance.

In this section, we present a position control scheme for our fingertip device. Since the device has $3 \mathrm{DoF}$, only three of the six parameters describing the platform configuration can be controlled. As common for these devices [3], [18], we choose to control the translation in the direction normal to the platform plane, $p_{z}$, together with its roll $\psi$ and pitch $\theta$ rotations (see Fig. 1). We collect these variables in the vector $\xi$. Therefore, when we control the device in position, the objective is to make the mobile platform follow a desired trajectory $\xi_{d}=$ $\left[p_{z d}, \psi_{d}, \theta_{d}\right]^{\mathrm{T}}$.

Two position control schemes are possible. In the first one, shown in Fig. 2a, the desired configuration $\xi_{d}$ is compared with the actual one $\xi$. Using an inverse kinematics procedure [16], [19], the error in the configuration space $\Delta \xi$ is transformed into an error in the input rotation space $\Delta q$, where $q=\left[q_{1}, q_{2}, q_{3}\right]^{\mathrm{T}}$ and the generic $q_{i}$ represent the rotation of the $i$-th proximal link, actuated by one of the three actuators (see Fig. 1). A PID controller is then used to define the torques $\tau=\left[\tau_{1}, \tau_{2}, \tau_{3}\right]$ to be applied by the device actuators. In this case, the device dynamics depends on the applied torques $\tau$ and on the resulting force and moment generated by the interaction with the fingertip, collected in the sixdimensional wrench $w$. Finally, rotational encoders on the actuators measure actual rotations $q$ of proximal links, and then, through a direct kinematic procedure, we evaluate the actual platform configuration. The main drawback of this

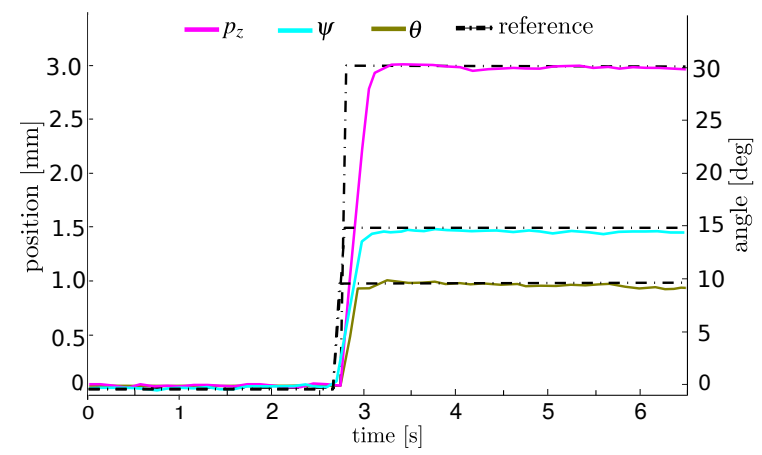

(a) Step response.

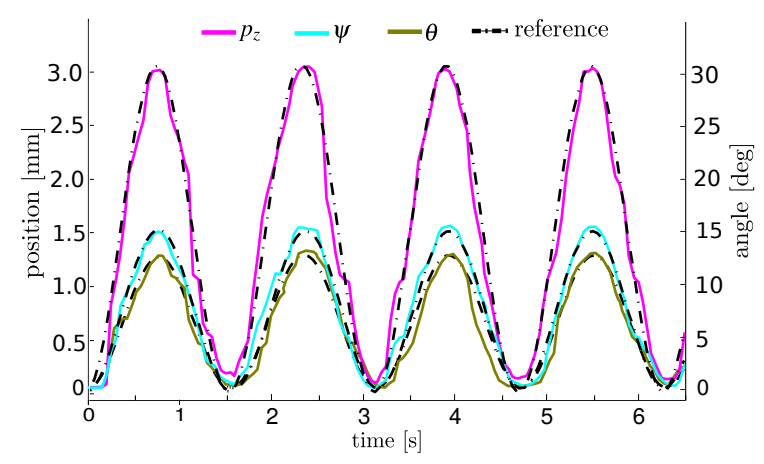

(b) Sine wave response.

Fig. 3. 3-DoF cutaneous device. Performance of the decoupled position control scheme shown in Fig. $2 b$.

approach is that it needs to solve the direct kinematic problem to estimate the platform configuration $\xi$ from the measured actuator rotations $q$. This estimation is not straightforward and it requires iterative procedures that may be computationally heavy.

In the second control scheme, shown in Fig. 2b, the desired configuration $\xi_{d}$ is directly transformed into reference values for the actuators $q_{d}$ through a standard inverse kinematics procedure [16]. The actual values $q$ are then directly measured by the encoders on the actuators. Finally, the torque applied by the motors is evaluated as a function of the error $\Delta q$, using a standard PID controller.

Evaluation. Fig. 3a shows the performance of the second control scheme, shown in Fig. 2b. We commanded a step signal as the desired position $\xi_{d}=\left[p_{z d}, \psi_{d}, \theta_{d}\right]^{\mathrm{T}}=$ $\left[3 \mathrm{~mm}, 15^{\circ}, 10^{\circ}\right]^{\mathrm{T}}$. Results show that the device reaches the desired position with a rise time of about $0.35 \mathrm{~s}$ and an error in the stationary phase lower than $2 \%$. The rise time is defined as the time required for the signal to rise from $10 \%$ to $90 \%$ of its steady value. Fig. $3 \mathrm{~b}$ shows the behavior of the device when the desired trajectory is sinusoidal: $p_{z d}(t)=1.5 \sin (4 t)+1.5 \mathrm{~mm}, \psi_{d}(t)=7.5 \sin (4 t)+7.5 \mathrm{deg}$, $\theta_{d}(t)=6 \sin (4 t)+6 \mathrm{deg}$.

After this, to quantitatively evaluate the performance error, we asked the device to simulate the contact with $n_{s}=100$ arbitrarily oriented surfaces. At each repetition, the system selected a random target platform configuration $\xi_{d, n}, n=$ $1, \ldots, 100$, with

$$
0 \leq p_{z d} \leq 4 \mathrm{~mm},-20^{\circ} \leq \psi_{d} \leq 20^{\circ},-20^{\circ} \leq \theta_{d} \leq 20^{\circ},
$$


For each repetition, we evaluated the error $\varepsilon_{n}=\xi_{d, n}-\xi_{a, n}$, where $\xi_{d, n}$ and $\xi_{a, n}$ indicate the $n$-th value of the desired and actual configurations, respectively, while $\xi_{a, n}$ is measured through the actuators' encoders and the direct kinematic procedure. For each sample, $\varepsilon_{n}=\left[e_{z, n}, e_{\psi, n}, e_{\theta, n}\right]^{T}$, where $\varepsilon_{z, n}$ is the position error in the $z$ direction, and $\varepsilon_{\psi, n}$ and $\varepsilon_{\theta, n}$ are the angular errors, according to the definition of the platform configuration vector $\xi$ previously introduced. The mean \pm std. deviation along all the repetitions of $\varepsilon_{z, n}, \varepsilon_{\psi, n}$, and $\varepsilon_{\theta, n}$, are $0.8 \pm 0.5 \mathrm{~mm}, 1.72 \pm 0.71^{\circ}$, and $1.14 \pm 0.29^{\circ}$ for $p_{z}, \psi$, and $\theta$, respectively. A Pearson's product-moment correlation is run to assess the relationship between the platform's position in the workspace and the above error. A preliminary analysis show that the relationship was linear with normally distributed variable, according to the Shapiro-Wilk test, and there are no outliers. There is no strong correlation between the platform position vs. rendering error $(|r|<0.3)$.

The same type of evaluation has also been carried out for the coupled control scheme shown in Fig. 2a. In this case, the mean error \pm std. deviation is $1.1 \pm 0.9 \mathrm{~mm}, 2.35 \pm 0.87^{\circ}$, and $1.72 \pm 0.56^{\circ}$ for $p_{z}, \psi$, and $\theta$, respectively. The same Pearson's product-moment correlation as above shows again no strong correlation between the platform position vs. rendering error.

\section{B. Cutaneous device force control}

Another way to control the fingertip device is to command a target force $f_{d}$ to be applied by the platform to the fingertip. To do this, let us assume that the mobile platform is equipped with a force sensor able to measure the contact force $f_{m}$.

We consider a force control loop with an internal position control loop, shown in Fig. 2c [20]. The desired contact force $f_{d}$ and the measured contact force $f_{m}$ are transformed into platform configurations by the compliance terms $C_{f d}$ and $C_{f m}$, respectively. The difference between such values is the desired platform configuration $\xi_{d}$, i.e.

$$
\xi_{d}=C_{f d} f_{d}-C_{f m} f_{m}
$$

The dimensions of $f_{d}$ and $f_{m}$ depend on the target application and sensing system. For example, if the platform is instrumented with a 3 -DoF force sensor, $f_{m} \in \mathbb{R}^{3}$. In this case, if the target force $f_{d}$ is also in $\mathbb{R}^{3}, C_{f d}$ and $C_{f m}$ are $3 \times 3$ matrices, e.g.,

$$
C_{f d}=C_{f m}=\left[\begin{array}{ccc}
0 & 0 & c_{\theta} \\
0 & c_{\psi} & 0 \\
c_{z} & 0 & 0
\end{array}\right],
$$

where $c_{z}$ is dimensionally a compliance, since it transforms the force error along $z$ in a reference displacement in the same direction, while $c_{\psi}$ and $c_{\theta}$ are rotations over a force. These compliance values depends on several parameters, including the user's finger dimension and mechanical characteristics [3]. In the literature [1], these values are often fixed and evaluated as an average of the human's fingertip skin compliance. In our prototype of Fig. 1, the platform is only instrumented with a 1DoF force sensor, which measures the contact force orthogonal to the platform surface. In this case, we consider a simplified form for $C_{f m}$, e.g., $C_{f m}=\left[c_{z}, 0,0\right]^{T}$. In other words, the force measured can be only used to regulate the reference value $p_{z d}$. In this case, $C_{f d}$ can be defined in different ways: we can choose again $C_{f d}=C_{f m}$, i.e., for the reference rotations we have $\psi_{d}=\theta_{d}=0$; or we can define $C_{f d}$ as a $3 \times 3$ matrix, as in eq. (2), i.e., we can set a three-dimensional force reference that is transformed by $C_{f d}$ in a reference configuration vector. This latter approach is the one adopted for the teleoperation experiment of Sec. IV.

The internal position control is similar to the one previously described and shown in Fig. 2b. In Fig. 2c, we also highlight the possibility of regulating the proportional term of the PID position controller, so as to simulate a platform desired stiffness $K_{d}$ [16]. In this case, it is necessary to evaluate the corresponding actuator stiffness matrix $K_{q}$, which depends both on the desired stiffness and the platform current configuration, according to the following relationship

$$
K_{q}=\tilde{J}^{\mathrm{T}} K_{d} \tilde{J} \delta q,
$$

where $\tilde{J}$ represents the device Jacobian matrix, which depends on device configuration and can be evaluated by analyzing the differential kinematics problem [19]. The torque $\tau$ is then evaluated as

$$
\tau=K_{q}\left(q_{d}-q\right) .
$$

Since $\tilde{J}$ depends on the platform's position and orientation, to simulate the interaction with a surface with a desired stiffness, $K_{q}$ needs to be evaluated at runtime, according to the current configuration. Moreover, a derivative term $D_{q}$ is present in the internal control loop so as to achieve a suitable damping in the system's response.

Evaluation. To quantitatively evaluate the performance of our device in rendering a certain target force $f_{d}=$ $\left[f_{x d}, f_{y d}, f_{z d}\right]^{T}$, we placed an ATI Mini 25 sensor (ATI Industrial Automation, Inc.) between the mobile platform and the upper body (i.e., where the fingertip should be). Then, we asked the mobile platform to apply $n_{s}=100$ random force vectors on the sensor. At each repetition, the system selected a random force vector $f_{d, n}, n=1, \ldots, 100$, with

$$
0 \leq f_{x d} \leq 2 \mathrm{~N}, 0 \leq f_{y d} \leq 2 \mathrm{~N}, 0 \leq f_{z d} \leq 4 \mathrm{~N} .
$$

Then, we compared each desired force $f_{d, n}$ with the actual force $f_{m, n}$, measured through the ATI sensor. The platform was held in each configuration for $1 \mathrm{~s}$, and the values gathered by the ATI were arithmetically averaged. The mean error \pm std. deviation is $0.32 \pm 0.14 \mathrm{~N}, 0.18 \pm 0.12 \mathrm{~N}$, and $0.43 \pm$ $0.19 \mathrm{~N}$ for $f_{x}, f_{y}$, and $f_{z}$ respectively. Similarly to Sec. II-A, a Pearson's product-moment correlation is run to assess the relationship between the platform's position in the workspace and the above error. There is no strong correlation between the platform position vs. rendering error $(|r|<0.3)$. Fig. 4 shows the performance of this control scheme.

\section{Exoskeleton position control with variable stiffness}

For the kinesthetic exoskeleton, we implemented a position control in which the stiffness can be varied. This control scheme is depicted in Fig. 5. 


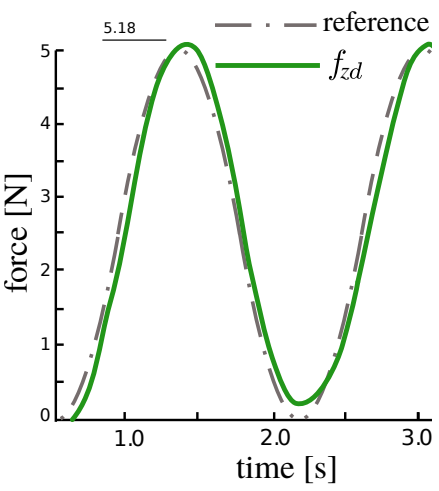

(a) Sine wave response.

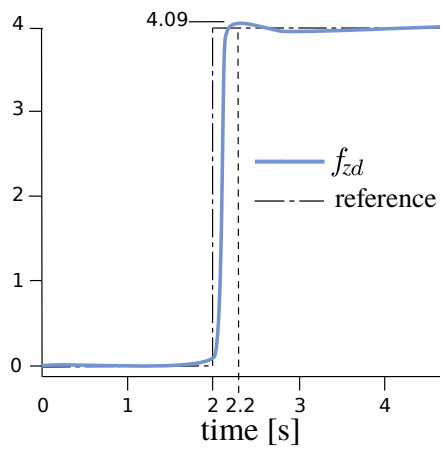

(b) Step response.
Fig. 4. 3-DoF cutaneous device. Performance of the force control scheme shown in Fig. 2c.

A desired rotation of the finger phalanx $\theta_{d}$ is set according to the considered interaction, i.e., to make the finger follow a certain trajectory (see Fig. 1). A straightforward kinematic analysis of the system composed by the exoskeleton and the user's finger allows to evaluate the corresponding value for the motor angle $\alpha_{d}$, that is then compared with the measure of the resolver. Finally, the error signal is used to evaluate the torque $\tau_{d}$ applied by the motor by means of a simple proportional control scheme

$$
\tau_{d}=k_{e}\left(\alpha_{d}-\alpha\right)
$$

where the proportional constant (stiffness) $k_{e}$ is varied according to the target interaction. A video showing the control of the exoskeleton with different $k_{e}$ can be found at https: //youtu.be/dw-o8nslgT0.

Evaluation. To quantitatively evaluate the performance of our exoskeleton in rendering a desired stiffness $k_{e d}$, we fixed the proximal clamp to a rigid support and the distal clamp to a dynamometer. Then, we applied a constant force to the distal clamp and we registered the actuator's displacement with different desired stiffness values $0 \leq k_{e d} \leq 2 \mathrm{Nmm} / \mathrm{rad}$. We ran $n_{s}=100$ iterations of this performance evaluation experiment. The exoskeleton was held in each configuration for $1 \mathrm{~s}$, and the values gathered by the potentiometer were arithmetically averaged. We registered a mean error \pm std. deviation of $0.67 \pm 0.10 \mathrm{Nmm} / \mathrm{rad}$.

\section{EXPERIMENT \#1: CURVATURE DISCRIMINATION}

To test the effectiveness of the proposed haptic device in rendering shapes, we carried out a curvature discrimination experiment. This experiment is inspired by the work of Prattichizzo et al. [3] and Frisoli et al. [8], who evaluated their fingertip systems using a similar approach. A video showing this curvature discrimination experiment can be found at https://youtu.be/_dKfbKOf14c.

\section{A. Experimental setup and participants}

Similarly to [3], [8], we used the same-different procedure to measure the just noticeable difference (JND) for curvature [21]. Six participants (4 males, 2 females, age range 24

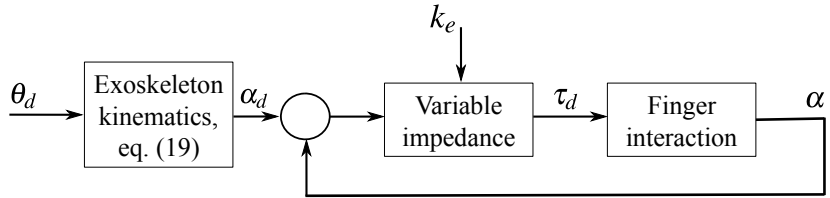

Fig. 5. Position control of the kinesthetic module.

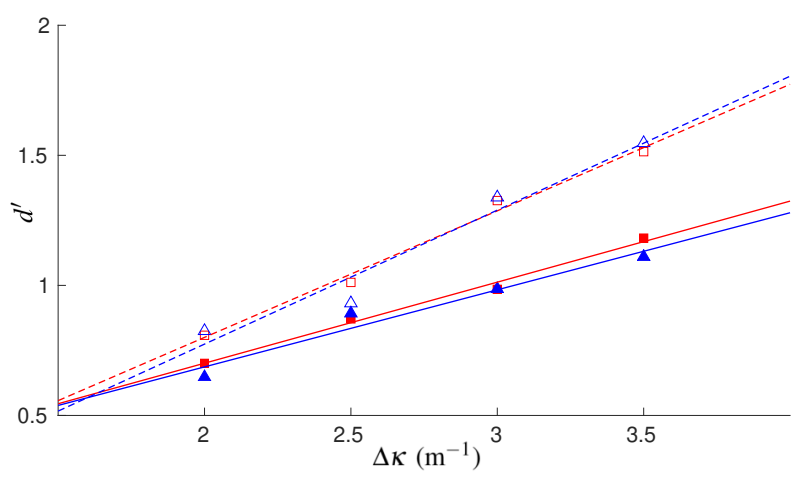

Fig. 6. Experiment \#1. Mean sensitivity measure $d^{\prime}$ vs. $\Delta \kappa$, together with the average interpolating lines for condition $\mathrm{K}$ (dashed lines), CK (solid lines), $\kappa_{b, 1}$ (blue lines), and $\kappa_{b, 2}$ (red lines).

- 33) took part to the experiment. One of them had previous experience with haptic interfaces. The experimental setup was composed of our complete wearable haptic device, shown in Fig. 1, and a Leap Motion tracking system.

\section{B. Experimental task and conditions}

Participants were blindfolded and asked to wear the proposed device on their right index finger. According to the aforementioned same-different procedure, participants were required to explore, in succession, two virtual spheres. The surface of each sphere was rendered at $20 \mathrm{~cm}$ from the table. The task consisted in judging if the curvature of the two virtual spheres was different or the same, relying on the feedback provided by the proposed wearable device. We registered the rate of correct responses given when the two spheres had different curvatures (hit rate, $p_{h}$ ) and the rate of incorrect responses when the two spheres had the same curvature (false alarm rate, $\left.p_{f}\right)[21]$.

We considered two different feedback conditions:

- Condition CK: kinesthetic and cutaneous feedback, provided by the complete device (3-DoF fingertip module + 1-DoF finger exoskeleton), and

- Condition K: kinesthetic feedback, provided by the 1DoF kinesthetic module only.

In condition $\mathrm{CK}$, both the cutaneous fingertip module and the kinesthetic finger module provide haptic cues to the subject. The fingertip display provides cutaneous feedback about the local geometry of the surface, according to the decoupled position control scheme described in Sec. II-A; while the finger exoskeleton provides kinesthetic force normal to the surface, according to the variable stiffness position control scheme described in Sec. II-C. In condition $\mathrm{K}$, only the kinesthetic finger module is active, while the 3-DoF mobile platform does not move. 


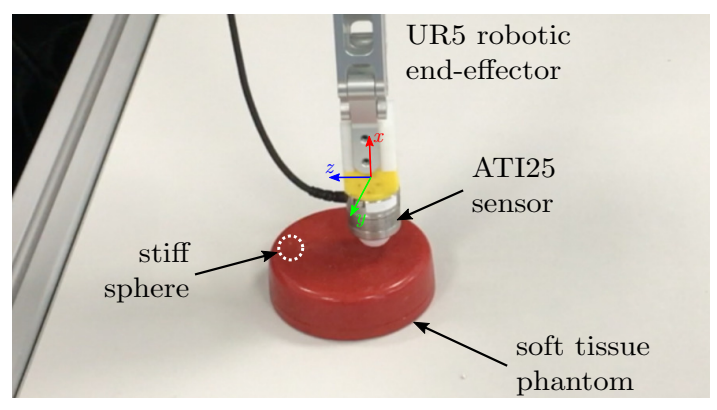

(a) Slave side.

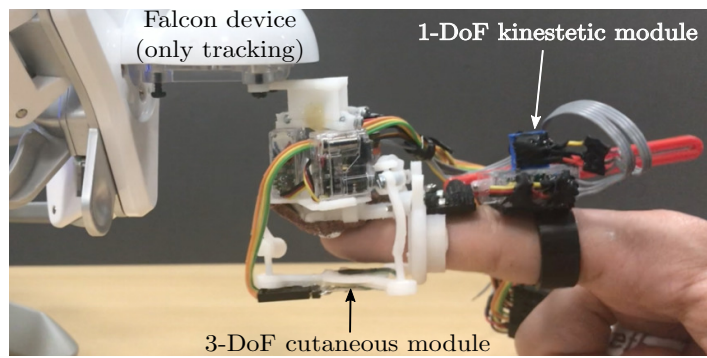

(b) Master side

Fig. 7. Experiment \#2: robot-assisted palpation. Setup. The slave system is composed of an ATI Nano25 sensor mounted on the endeffector of a 6-DoF Universal Robot UR5 manipulator. The master system is composed of our complete wearable haptic device attached to the end-effector of a Novint Falcon device. The Falcon is only used to track the position of the user's finger and does not provide any force feedback. The remote environment is composed of a simulated prostate tissue model, with a 1-cm-diameter stiff sphere embedded at $3 \mathrm{~mm}$ from the surface.

Each subject carried out eight series of trials, in which spheres with different curvature values, $\kappa_{a, *}$ and $\kappa_{b, \star}(\Delta \kappa=$ $\left.\kappa_{b, \star}-\kappa_{a, *}\right)$, were taken into account:

(i) $\kappa_{a, 1}=3.5 \mathrm{~m}^{-1}$ and $\kappa_{b, 1}=7 \mathrm{~m}^{-1}\left(\Delta \kappa=3.5 \mathrm{~m}^{-1}\right)$,

(ii) $\kappa_{a, 2}=4 \mathrm{~m}^{-1}$ and $\kappa_{b, 1}=7 \mathrm{~m}^{-1}\left(\Delta \kappa=3 \mathrm{~m}^{-1}\right)$,

(iii) $\kappa_{a, 3}=4.5 \mathrm{~m}^{-1}$ and $\kappa_{b, 1}=7 \mathrm{~m}^{-1}\left(\Delta \kappa=2.5 \mathrm{~m}^{-1}\right)$,

(iv) $\kappa_{a, 4}=5 \mathrm{~m}^{-1}$ and $\kappa_{b, 1}=7 \mathrm{~m}^{-1}\left(\Delta \kappa=2 \mathrm{~m}^{-1}\right)$,

(v) $\kappa_{a, 5}=1.5 \mathrm{~m}^{-1}$ and $\kappa_{b, 2}=5 \mathrm{~m}^{-1}\left(\Delta \kappa=3.5 \mathrm{~m}^{-1}\right)$,

(vi) $\kappa_{a, 6}=2 \mathrm{~m}^{-1}$ and $\kappa_{b, 2}=5 \mathrm{~m}^{-1}\left(\Delta \kappa=3 \mathrm{~m}^{-1}\right)$,

(vii) $\kappa_{a, 7}=2.5 \mathrm{~m}^{-1}$ and $\kappa_{b, 2}=5 \mathrm{~m}^{-1}\left(\Delta \kappa=2.5 \mathrm{~m}^{-1}\right)$.

(viii) $\kappa_{a, 8}=3 \mathrm{~m}^{-1}$ and $\kappa_{b, 2}=5 \mathrm{~m}^{-1}\left(\Delta \kappa=2 \mathrm{~m}^{-1}\right)$.

Each series consists of 24 repetitions of the curvature discrimination task, with 12 trials for each feedback condition. The entire experiment lasted approximately 60 minutes.

On each repetition of each series, two spheres with random curvature $\left(\kappa_{a, *}\right.$ or $\left.\kappa_{b}\right)$ were rendered. In this way, the probability of exploring a pair of spheres with the same (different) curvature was 0.5 . To minimize learning effects, the order of presentation of the series and feedback conditions was randomized.

\section{Results}

False alarm and hit rates were recorded for each subject and converted to $z$ scores of the normal distribution [21], [22]. According to the criterion already adopted in [3], [8], [21], we evaluated the discrimination threshold as the difference between the curvatures for which $d^{\prime}=z_{h}-z_{f}=1$, where $d^{\prime}$ is the so-called "sensitivity index" [21]. The JND was then computed for each subject and for each condition CK and $\mathrm{K}$, assuming $d^{\prime}$ to increase linearly. Finally, the overall JND was computed as the average of the JND values of all the participants. Fig. 6 shows the mean sensitivity measure $d^{\prime}$ vs. $\Delta \kappa$ and the average interpolating lines for condition $\mathrm{K}$ (dashed lines), CK (solid lines), $\kappa_{b, 1}$ (blue lines), and $\kappa_{b, 2}$ (red lines). A Shapiro-Wilk normality test confirmed the normality of the registered data. A parametric two-tailed paired t-test showed that the average JND values were significantly lower $(t=7.633, p=0.001$ ) for condition CK than for $\mathrm{K}$, with an average \pm std. of $2.41 \pm 0.11 \mathrm{~m}^{-1}$ and $3.00 \pm 0.16 \mathrm{~m}^{-1}$ for conditions $\mathrm{CK}$ and $\mathrm{K}$, respectively. Time needed to complete the given task was recorded as well. No statistical difference was found between the two conditions for this metric.

Results show that the combination of cutaneous and kinesthetic feedback leads to better performance than employing solely kinesthetic feedback. These results confirm the effectiveness of the proposed cutaneous/kinesthetic solution and that the cutaneous display of surface orientation helps the haptic perception of shape. The discrimination threshold for curvature observed in this work is in fact in agreement with previous results in the literature [3], [8]. An interesting work analyzing the role of kinesthetic and cutaneous cues in curvature discrimination can be found in [23].

At the end of this experiment, we asked the participants to rate the perceived effectiveness of the two feedback conditions, along with the comfort and level of wearability of the proposed haptic system. The responses were given using a slider that ranged from 0 to 10 , where a score of 0 meant "very low" and a score of 10 meant "very high". Wearability of the system was rated 8.2 out of 10 and the comfort of the system was rated 7.7 out of 10 . The perceived effectiveness of conditions CK and K were rated 8.0 and 6.7 out of 10, respectively.

\section{EXPERIMENT \#2: ROBOT-ASSISTED PALPATION}

To evaluate the effectiveness and viability of our haptic system in a robotic scenario, we carried out a robot-assisted palpation task enrolling 20 human subjects. This experiment is inspired by the work of Pacchierotti et al. [5], who evaluated their cutaneous fingertip device in a palpation experiment using a da Vinci Surgical System. A video showing this palpation experiment can be found at https://youtu.be/P2oABhEcBUI.

\section{A. Experimental setup and participants}

Fig. 7 show the experimental setup. The slave system is composed of a six-axis ATI Nano25 force sensor mounted on the end-effector of a 6-DoF Universal Robot UR5 manipulator. It has high speed output, span temperature compensation, and signal-to-noise ratio. The master side is composed of our complete wearable haptic device attached to the end-effector of a Novint Falcon device. The Falcon is a 3-DoF parallel grounded haptic interface, but here it is only used to track the position of the user's finger, and it did not provide any force feedback. The remote environment is composed of a simulated prostate tissue model made from Ecoflex 0010 (Smooth-On Inc., USA) and brown dye. To simulate the presence of a 


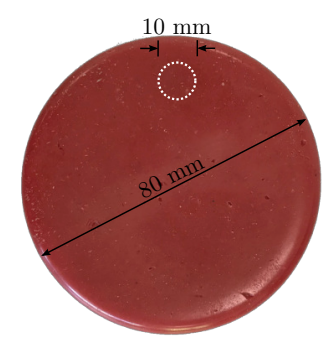

(a) Top view

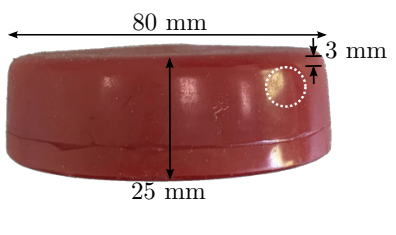

(b) Side view

Fig. 8. Simulated prostate tissue model. The overall diameter and thickness of the model are $80 \mathrm{~mm}$ and $25 \mathrm{~mm}$, respectively. A $1-\mathrm{cm}$ diameter plastic sphere is embedded at a depth of $3 \mathrm{~mm}$ from the surface to mimic a tumor.

tumor [24], a stiff plastic sphere with a diameter of $1 \mathrm{~cm}$ is embedded into the tissue model at $3 \mathrm{~mm}$ from the surface (see Fig. 8). The plastic sphere is not visible from the outside. To minimize the utility of any incidental visual cues and to reduce any learning effect, the study used four copies of the prostate model, that were interchanged between trials. The location of the stiff sphere changed across the different copies, but its depth was kept constant at $3 \mathrm{~mm}$ from the surface.

Twenty participants took part in the experiment, including 5 women and 15 men. Seven of them had previous experience with haptic interfaces. The experimenter explained the procedures and spent about five minutes adjusting the setup to be comfortable before the subject began the experiment. Each subject then spent about two minutes practicing controlling the slave robot through the master interface.

\section{B. Implementation of the robotic teleoperation system}

The teleoperation system was managed by a GNU/Linux machine (Ubuntu 16.04 with Linux Kernel 4.4).

We use the Novint Falcon to measure the position of the subject's finger and set the one of the slave end-effector. The Falcon does not provide any force feedback. The velocities of the robotic manipulator joints $\dot{q}_{r} \in \mathbb{R}^{6}$ are then commanded as

$$
\dot{q}_{r}=J_{r}^{\#} \dot{p}_{h} v_{m}
$$

where $J_{r} \in \mathbb{R}^{3 \times 6}$ represents the manipulator Jacobian matrix [20], apex \# indicates its pseudo-inverse, $\dot{p}_{h} \in \mathbb{R}^{3}$ are the velocities of the haptic interface's end-effector, and $v_{m} \in \mathbb{R}$ is the scaling factor between the master and slave workspaces. Scaling factor $v_{m}$ is tunable to enable coarse and fine gestures. In this experiment, $v_{m}$ was set to 1.3 in order to enable the subject to explore all the surface of the tissue phantom without clutching.

The 3-DoF haptic interface registers the motion of the operator at $500 \mathrm{~Hz}$. The Universal Robot manipulator then follows the motion commanded by the haptic interface at $125 \mathrm{~Hz}$, according to eq. (6). Finally, the ATI force/torque sensor registers interaction forces at the slave side at $1 \mathrm{kHz}$. Since cutaneous feedback does not affect the stability of the teleoperation loop, we did not implement any specific stability controller for conditions N and C [13], [25]. On the other hand, the stability of condition $\mathrm{CK}$ was guaranteed by the passivity controller described in [26], [27].

\section{Experimental task and conditions}

The task consisted of exploring the tissue model to try to detect the location of the hidden plastic sphere. The task started when the Nano25 sensor touched the tissue model for the first time and ended when the subject told the experimenter that he found the hidden sphere. Each participant performed twelve trials of the palpation task, with four repetitions for each of the following three feedback conditions:

- Condition N: no haptic feedback,

- Condition C: cutaneous feedback only provided by the 3-DoF fingertip module,

- Condition CK: cutaneous and kinesthetic feedback provided by the complete device.

In all conditions, the user was asked to wear the complete wearable device as shown in Fig. 7b. In condition $\mathrm{N}$, the cutaneous and kinesthetic modules were not active. The mobile platform was always in contact with the subject's fingertip and the kinesthetic module was driven to apply no force to the subject's finger. In condition $\mathrm{C}$, the cutaneous module provided cutaneous stimuli to render the interaction with the tissue phantom, according to the force control scheme described in Sec. II-B. Again, the kinesthetic module was driven to apply no force to the subject's finger. In condition CK, both the cutaneous and kinesthetic modules provided cutaneous and kinesthetic stimuli, respectively, to render the interaction with the tissue phantom, according to the control schemes described in Secs. II-B and II-C. The slave system was placed at $1 \mathrm{~m}$ from the master console, so that subjects were always able to see the operative environment.

Each subject performed all four repetitions of a single feedback condition as a block, and the order of the conditions was randomized to test all six possible combinations exactly three times. At the end of each condition, each subject was asked to rate, on two sliders going from 0 to 10 , "how easy was it to detect the location of the stiff sphere?" and "how confident were you in detecting the location of the stiff sphere?" A score of 0 meant "very difficult" or "not at all confident," and a score of 10 meant "very easy" or "very confident." At the end of the experiment, each subject was asked to choose which feedback conditions were the most and least effective at enabling detection of the sphere's position.

\section{Results}

To evaluate the subject's performance under each of the considered feedback conditions, we evaluated (1) the absolute error in detecting the location of the plastic sphere, (2) the task completion time, and (3) the root mean square (RMS) pressure exerted by the Nano25 sensor on the tissue model. A low value of these three metrics denotes the best performance.

Fig. 9a shows the absolute position error results for the three experimental conditions. The position error is calculated as the planar distance between the location indicated by the subject on the phantom surface and the projection of the center of the sphere on the same surface. A repeated-measures ANOVA showed a statistically significant difference between the means of the three feedback conditions $\left(\mathrm{F}_{2,38}=9.245\right.$, 


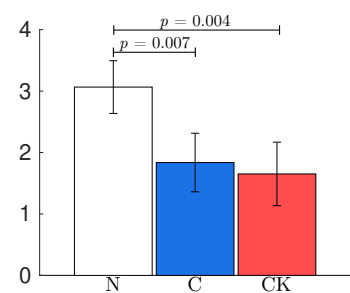

(a) Error in locating the stiff sphere $(\mathrm{cm})$.

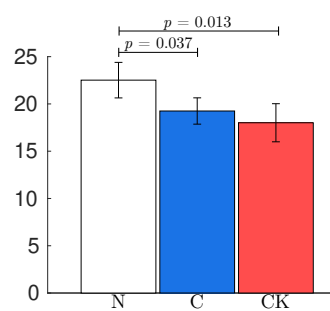

(b) Completion time (s).

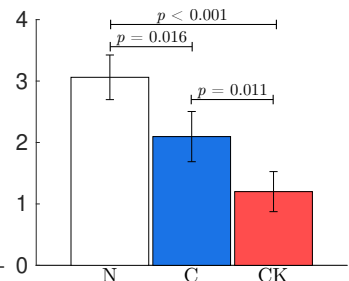

(c) RMS force $(\mathrm{N})$

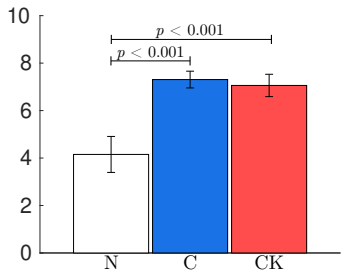

(d) "how easy..."

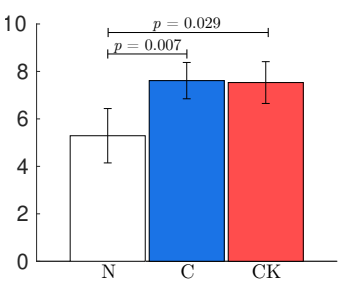

(e) "how confident..."

Fig. 9. Experiment \#2: robot-assisted palpation. Mean and 95\% confidence interval of the (a) error in locating the position of the sphere, (b) completion time, (c) RMS force applied by the slave robot on the tissue phantom, and preference ratings for questions (d) "how easy was it to detect the location of the stiff sphere?" and (e) "how confident were you in detecting the location of the stiff sphere?" are plotted. P-values of post-hoc group comparisons are reported when statistically different.

$p=0.001, \mathrm{a}=0.05)$. Post-hoc analysis (Games-Howell posthoc test) revealed statistically significant differences between conditions $\mathrm{N}$ and $\mathrm{C}(p=0.007)$ and between $\mathrm{N}$ and $\mathrm{CK}$ $(p=0.004)$. Fig. 9b shows the completion time results. The collected data passed the Shapiro-Wilk normality test and Mauchly's Test of Sphericity. A repeated-measures ANOVA showed a statistically significant difference between the means of the three feedback conditions $\left(\mathrm{F}_{2,38}=6.132, p=0.005\right.$, $\mathrm{a}=0.05)$. Post-hoc analysis (Games-Howell post-hoc test) revealed statistically significant differences between conditions $\mathrm{N}$ and $\mathrm{C}(p=0.037)$ and between $\mathrm{N}$ and CK $(p=0.013)$. Fig. 9c shows the RMS force exerted by the slave end-effector on the tissue model, registered as the norm of the 3-DoF force sensed by the ATI Nano25. A repeated-measures ANOVA showed a statistically significant difference between the means of the three feedback conditions $\left(\mathrm{F}_{2,38}=22.922, p<0.001\right.$, $\mathrm{a}=0.05)$. Post-hoc analysis (Games-Howell post-hoc test) revealed statistically significant differences between conditions $\mathrm{N}$ and $\mathrm{C}(p=0.016), \mathrm{N}$ and $\mathrm{CK}(p<0.001)$, and $\mathrm{C}$ and $\mathrm{CK}$ $(p=0.011)$.

Finally, we analyzed the ratings given by the subjects at the end of each feedback condition. Fig. 9d shows the ratings that the three feedback conditions received for the first question ("how easy"). A repeated-measures ANOVA showed a statistically significant difference between the means of the three feedback conditions $\left(\mathrm{F}_{2,38}=44.707, p<0.001, \mathrm{a}=0.05\right)$. Post-hoc analysis (Games-Howell post-hoc test) revealed statistically significant differences between conditions $\mathrm{N}$ and $\mathrm{C}$ $(p<0.001)$ and between $\mathrm{N}$ and CK $(p<0.001)$. Fig. 9e shows the ratings that the three feedback conditions received for the second question ("how confident"). A repeated-measures ANOVA showed a statistically significant difference between the means of the three feedback conditions $\left(\mathrm{F}_{2,38}=8.034\right.$, $p=0.001, \mathrm{a}=0.05$ ). Post-hoc analysis (Games-Howell posthoc test) revealed statistically significant differences between conditions $\mathrm{N}$ and $\mathrm{C}(p=0.007)$ and between $\mathrm{N}$ and $\mathrm{CK}$ $(p=0.029)$. Fourteen subjects chose condition $\mathrm{CK}$ as the most effective feedback condition, four subjects chose condition $\mathrm{C}$, and only two chose condition $\mathrm{N}$. The two outlier subjects who chose condition $\mathrm{N}$ said that they considered the haptic feedback useless.

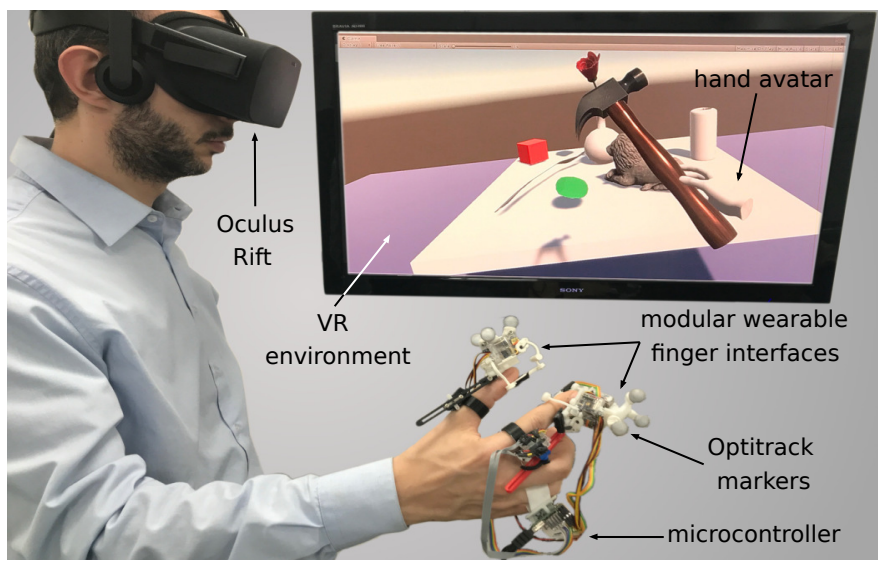

Fig. 10. Experimental setup. Subjects were asked to interact with the virtual environment while wearing two wearable cutaneous devices, one on the thumb and one on the index finger. The virtual environment is composed of seven different objects lying on a table. Interaction forces in the virtual environment are provided to the user by the cutaneous device as indicated in Sec. II.

\section{EXPERIMENT \#3: INTERACTION IN A VIRTUAL REALITY (VR) ENVIRONMENT}

Finally, we carried out a third experiment in a virtual reality environment. This experience has been inspired by the experiments carried out in [16], [28], [29], [30].

\section{A. Experimental setup and participants}

Fig. 10 shows the experimental setup. It is composed of our complete wearable haptic device and a virtual environment with 7 different objects. Fourteen participants (10 males, 4 female, age range 25 - 30) took part to the experiment, all of whom were right-handed. Eight of them had previous experience with haptic interfaces.

\section{B. Experimental task and conditions}

Users are asked to wear two prototypes of our complete haptic devices on the thumb and index fingers of their right hand, and interact with the virtual environment. The users hand pose is tracked using an Optitrack system, and a virtual hand mimicked the subjects hand pose in the virtual environment. Every time one of the virtual fingers comes in contact with a 
TABLE I

EXPERIMENT \#3. USE QUESTIONNAIRE RESULTS.

\begin{tabular}{|c|c|c|c|}
\hline QA. Usefulness & $\mathbf{N}$ & C & CK \\
\hline 1. It helps me be more effective & $7.0 \pm 2.8$ & $11.0 \pm 1.3$ & $12.7 \pm 1.8$ \\
\hline $\begin{array}{l}\text { 2. It helps me be more produc- } \\
\text { tive }\end{array}$ & $6.1 \pm 1.9$ & $10.1 \pm 1.8$ & $11.7 \pm 1.9$ \\
\hline 3. It is useful & $10.5 \pm 1.2$ & $12.1 \pm 1.3$ & $12.8 \pm 1.9$ \\
\hline $\begin{array}{l}\text { 4. It makes the things I want to } \\
\text { accomplish easier to get done }\end{array}$ & $5.3 \pm 2.3$ & $9.5 \pm 1.8$ & $11.0 \pm 2.2$ \\
\hline \multicolumn{4}{|l|}{ QB. Ease of use } \\
\hline 1. It is easy to use & $12.0 \pm 1.3$ & $10.0 \pm 1.0$ & $7.1 \pm 1.5$ \\
\hline 2. It is simple to use & $11.8 \pm 1.2$ & $9.8 \pm 1.1$ & $6.0 \pm 2.3$ \\
\hline 3. It is user friendly & $12.6 \pm 1.5$ & $11.1 \pm 1.5$ & $10.9 \pm 2.7$ \\
\hline 4. Using it is effortless & $12.3 \pm 1.6$ & $10.0 \pm 1.1$ & $6.6 \pm 2.4$ \\
\hline $\begin{array}{l}\text { 5. I can use it without written } \\
\text { instructions }\end{array}$ & $12.7 \pm 1.5$ & $13.1 \pm 1.2$ & $11.7 \pm 1.5$ \\
\hline $\begin{array}{l}\text { 6. I do not notice any inconsis- } \\
\text { tencies as I use it }\end{array}$ & $12.8 \pm 1.4$ & $12.1 \pm 1.2$ & $12.4 \pm 1.4$ \\
\hline $\begin{array}{l}\text { 7. Both occasional and regular } \\
\text { users would like it }\end{array}$ & $8.7 \pm 2.0$ & $10.8 \pm 1.1$ & $13.4 \pm 0.8$ \\
\hline $\begin{array}{l}\text { 8. I can recover from mistakes } \\
\text { quickly and easily }\end{array}$ & $11.8 \pm 1.8$ & $12.7 \pm 1.1$ & $11.8 \pm 1.8$ \\
\hline $\begin{array}{l}\text { 9. I can use it successfully ev- } \\
\text { ery time }\end{array}$ & $13.6 \pm 0.8$ & $10.1 \pm 1.6$ & $10.3 \pm 1.5$ \\
\hline \multicolumn{4}{|l|}{ QC. Ease of learning } \\
\hline 1. I learned to use it quickly & $11.8 \pm 2.2$ & $12.1 \pm 2.1$ & $12.4 \pm 1.8$ \\
\hline 2. It is easy to learn to use it & $10.5 \pm 1.9$ & $9.4 \pm 1.4$ & $9.4 \pm 1.8$ \\
\hline $\begin{array}{l}\text { 3. I quickly became skillful } \\
\text { with it }\end{array}$ & $9.2 \pm 1.7$ & $8.4 \pm 0.9$ & $8.6 \pm 1.3$ \\
\hline \multicolumn{4}{|l|}{ QD. Satisfaction } \\
\hline 1. I am satisfied with it & $6.8 \pm 1.8$ & $9.7 \pm 1.2$ & $12.8 \pm 1.6$ \\
\hline 2. It is fun to use & $10.0 \pm 1.3$ & $11.4 \pm 1.0$ & $13.1 \pm 0.9$ \\
\hline $\begin{array}{l}\text { 3. It works the way I want it to } \\
\text { work }\end{array}$ & $11.8 \pm 1.6$ & $13.5 \pm 1.1$ & $14.1 \pm 0.7$ \\
\hline 4. It is wonderful & $5.7 \pm 1.4$ & $9.0 \pm 1.2$ & $10.8 \pm 1.1$ \\
\hline 5. It is pleasant to use & $10.8 \pm 1.9$ & $10.6 \pm 1.9$ & $11.8 \pm 2.3$ \\
\hline
\end{tabular}

virtual object, the haptic device applies haptic stimuli to the corresponding finger. The task consists of interacting with the different virtual objects for 7 minutes (e.g., picking them up, poking them, squeezing them, pushing them, throwing them around). We considered the same feedback modalities as in Sec. IV-C, i.e.,

- Condition N: no haptic feedback,

- Condition C: cutaneous feedback only provided by the 3-DoF fingertip module,

- Condition CK: cutaneous and kinesthetic feedback provided by the complete device.

Interaction forces in the virtual environment were computed using a virtual proxy algorithm [31] and they were provided by the haptic device as indicated in Secs. II-B and II-C. This algorithm uses a proxy point attached to the haptic interaction point by a virtual spring (representing the surface stiffness).
TABLE II

EXPERIMENT \#3. STATISTICAL ANALYSIS.

\begin{tabular}{|l|c|c|c|}
\hline Questions & N vs. C & N vs. CK & C vs. CK \\
\hline QA.1 & $p<0.001$ & $p<0.001$ & $p=0.042$ \\
\hline QA.2 & $p<0.001$ & $p<0.001$ & $p=0.027$ \\
\hline QA.3 & $p=0.005$ & $p=0.011$ & - \\
\hline QA.4 & $p=0.001$ & $p<0.001$ & $p=0.012$ \\
\hline QB.1 & $p=0.001$ & $p<0.001$ & $p<0.001$ \\
\hline QB.2 & $p=0.004$ & $p<0.001$ & $p<0.001$ \\
\hline QB.4 & $p=0.003$ & $p<0.001$ & $p=0.001$ \\
\hline QB.7 & $p=0.025$ & $p<0.001$ & $p=0.001$ \\
\hline QB.9 & $p<0.001$ & $p<0.001$ & - \\
\hline QD.1 & $p<0.001$ & $p<0.001$ & $p<0.001$ \\
\hline QD.2 & $p=0.043$ & $p<0.001$ & $p=0.001$ \\
\hline QD.3 & $p=0.023$ & $p=0.001$ & - \\
\hline QD.4 & $p<0.001$ & $p<0.001$ & $p=0.002$ \\
\hline
\end{tabular}

When the haptic interaction point moves within a virtual object, the proxy point is constrained to the object surface, stretching the spring and defining a virtual interaction force.

\section{Results}

We evaluated the immersiveness of the haptic-enabled virtual reality scenario through the Usefulness, Satisfaction, and Ease of use (USE) questionnaire [32]. At the end of each condition, subjects were asked to rate their experience on a slider going from 0 to 15 , where a score of 15 was described as "completely agree" and a score of 1 as "completely disagree" with the assertion. Similarly to [16], our USE questionnaire is composed of 21 questions. Questions and answers for each condition are reported in Table I. To determine whether the answers registered differ between the three feedback conditions, we ran twenty-one repeated-measures ANOVA tests (significance level alpha $=0.05$ ), one for each question. Results of this statistical analysis are reported in Table II.

We also asked which condition the subjects preferred. Six subjects preferred condition $\mathrm{CK}$, three condition $\mathrm{C}$, and one condition $\mathrm{N}$.

\section{Vi. Discussion, Conclusion and Perspectives}

In this paper, we presented the control and evaluation of a novel wearable modular 4-DoF exoskeleton for haptic interaction. The device is composed of a 3-DoF fingertip cutaneous device and a 1-DoF finger exoskeleton, which can be either used together as a single device or separately as two different devices. It weighs only $42 \mathrm{~g}$ for $117 \times 50 \times 43 \mathrm{~mm}$ dimensions. With respect to other wearable solutions presented in the literature, the proposed device is extremely compact and lightweight, and it features a quite unique modular structure. Moreover, the 3-DoF fingertip module solves the indeterminacy due to the underactuation of the platform, and the 1DoF finger module has been designed to be easily adjusted to different finger sizes. 
To test its effectiveness and wearability, we carried out three experimental evaluations, enrolling a total of 40 human participants. The first experiment tested the capability of our device in differentiating objects with different curvatures. Results showed that providing both cutaneous and kinesthetic feedback (CK) led to a JND significantly lower than providing only kinesthetic feedback $(\mathrm{K})$. Therefore, users were able to better differentiate the curvatures when also provided with cutaneous information. All subjects found our system wearable, comfortable, and easy to wear. All of them were able to don the device with minimal assistance. It is interesting to notice that the tracking of the hand worked quite well even using a Leap Motion sensor. This may seem quite surprising, as the exoskeleton significantly occludes the user's hand. However, we believe that the Leap Motion still managed to achieve a satisfactory tracking thanks to the fact that users kept their hand mostly open.

The second experiment considered a robot-assisted palpation task. Providing haptic feedback (either $\mathrm{C}$ or $\mathrm{CK}$ ) led to a significantly better performance with respect to not providing any force feedback $(\mathrm{N})$ in all the considered metrics. Moreover, providing both kinesthetic and cutaneous feedback (CK) did not significantly reduce the error in locating the sphere or the completion time with respect to providing cutaneous feedback only (C). This result means that, for this task, providing additional kinesthestic feedback was not particularly useful, and that the local geometry information provided by the 3DoF cutaneous module was already sufficient. However, in condition $\mathrm{CK}$, users exerted significantly less force than in condition $\mathrm{C}$, which is quite important, as applying excessive force when manipulating tissue may lead to significant damage [33]. Finally, we also evaluated the user's preference when using the system, registering preference ratings for questions "how easy was it to detect the location of the stiff sphere?" and "how confident were you in detecting the location of the stiff sphere?". Providing haptic feedback (either C or CK) led to significantly better ratings with respect to not providing any force feedback $(\mathrm{N})$. As before, providing both kinesthetic and cutaneous feedback (CK) did not produce significantly higher ratings with respect to providing cutaneous feedback only (C). These results are in agreement with the experiment carried out by [5]. Also there, providing cutaneous feedback during a robot-assisted palpation task led to improved performance with respect to providing no haptic feedback at all. However, the device used in [5] did not provide any kinesthetic feedback and it was not designed to be wearable/portable. In this second experiment, to precisely track the position of the fingers, we used a Falcon interface, which guaranteed a high tracking accuracy (i.e., more than 10 times higher than the Leap Motion used in the first experiment). However, of course, this choice severely reduced the workspace of the system, and it did not enable us to evaluate again the portability and wearability of our system. Moreover, the Falcon interfaces, even when commanded to provide no external force, shows an internal stiffness and friction that is unwittingly displayed to the user. Nonetheless, since this effect was present in all the considered conditions, we expect it to have negligible effect in the final computation of the considered metrics.
The third and last experiment considered a Virtual Reality experiment. Users were asked to report on their experience through a 21-question Usefulness, Satisfaction, and Ease of use (USE) questionnaire, as well as to choose their preferred condition. In most questions, condition CK performed the best, followed by condition $\mathrm{C}$ and then by condition N. Similarly, most subjects preferred condition CK.

In the near future, we will work on extending the experimental evaluation, testing a larger set of feedback conditions, scenarios, and applications, enrolling an even higher number of participants. Finally, we will also study how the control of the platform should be adjusted for different finger shapes and sizes.

\section{REFERENCES}

[1] C. Pacchierotti, S. Sinclair, M. Solazzi, A. Frisoli, V. Hayward, and D. Prattichizzo, "Wearable haptic systems for the fingertip and the hand: Taxonomy, review, and perspectives," IEEE Trans. Haptics, vol. 10, no. 4, pp. 580-600, 2017.

[2] K. Minamizawa, S. Fukamachi, H. Kajimoto, N. Kawakami, and S. Tachi, "Gravity grabber: wearable haptic display to present virtual mass sensation," in Proc. ACM Special Interest Group on Computer Graphics and Interactive Techniques, 2007, pp. 8-es.

[3] D. Prattichizzo, F. Chinello, C. Pacchierotti, and M. Malvezzi, "Towards wearability in fingertip haptics: a 3-DoF wearable device for cutaneous force feedback," IEEE Trans. Haptics, vol. 6, no. 4, pp. 506-516, 2013.

[4] X. De Tinguy, C. Pacchierotti, M. Marchal, and A. Lecuyer, "Enhancing the stiffness perception of tangible objects in mixed reality using wearable haptics," in Proc. IEEE Conf. Virtual Reality, 2018.

[5] C. Pacchierotti, D. Prattichizzo, and K. J. Kuchenbecker, "Cutaneous feedback of fingertip deformation and vibration for palpation in robotic surgery," IEEE Trans. Biomedical Eng., vol. 63, pp. 278-287, 2016.

[6] B. B. Edin, L. Ascari, L. Beccai, S. Roccella, J.-J. Cabibihan, and M. Carrozza, "Bio-inspired sensorization of a biomechatronic robot hand for the grasp-and-lift task," Brain research bulletin, vol. 75, no. 6, pp. 785-795, 2008.

[7] K. Kuchenbecker, D. Ferguson, M. Kutzer, M. Moses, and A. Okamura, "The touch thimble: Providing fingertip contact feedback during pointforce haptic interaction," in Proc. Symp. on Haptic interfaces for virtual environment and teleoperator systems, 2008, pp. 239-246.

[8] A. Frisoli, M. Solazzi, F. Salsedo, and M. Bergamasco, "A fingertip haptic display for improving curvature discrimination," Presence: Teleoperators and Virtual Environments, vol. 17, no. 6, pp. 550-561, 2008.

[9] A. Girard, M. Marchal, F. Gosselin, A. Chabrier, F. Louveau, and A. Lécuyer, "Haptip: Displaying haptic shear forces at the fingertips for multi-finger interaction in virtual environments," Frontiers in ICT, vol. 3, p. 6, 2016

[10] M. Cempini, M. Cortese, and N. Vitiello, "A powered finger-thumb wearable hand exoskeleton with self-aligning joint axes," IEEE/ASME Trans. Mechatronics, vol. 20, no. 2, pp. 705-716, 2015.

[11] M. Sarac, M. Solazzi, D. Leonardis, E. Sotgiu, M. Bergamasco, and A. Frisoli, "Design of an underactuated hand exoskeleton with joint estimation," in Adv. Italian Mechanism Science, 2017, pp. 97-105.

[12] D. Wang, M. Song, A. Naqash, Y. Zheng, W. Xu, and Y. Zhang, "Toward whole-hand kinesthetic feedback: A survey of force feedback gloves," IEEE Transactions on Haptics, 2018.

[13] C. Pacchierotti, L. Meli, F. Chinello, M. Malvezzi, and D. Prattichizzo, "Cutaneous haptic feedback to ensure the stability of robotic teleoperation systems," The Int. Journal of Robotics Research, vol. 34, no. 14, pp. 1773-1787, 2015.

[14] L. Meli, C. Pacchierotti, and D. Prattichizzo, "Sensory subtraction in robot-assisted surgery: fingertip skin deformation feedback to ensure safety and improve transparency in bimanual haptic interaction," IEEE Trans. Biomedical Engineering, vol. 61, no. 4, pp. 1318-1327, 2014.

[15] Z. F. Quek, W. Provancher, and A. Okamura, "Evaluation of skin deformation tactile feedback for teleoperated surgical tasks," IEEE Transactions on Haptics, 2018.

[16] F. Chinello, C. Pacchierotti, M. Malvezzi, and D. Prattichizzo, "A three revolute-revolute-spherical wearable fingertip cutaneous device for stiffness rendering," IEEE Trans. Haptics, vol. 11, no. 1, pp. 39-50, 2018. 
[17] S. Lemerle, T. Nozaki, and K. Ohnishi, "Design and evaluation of a remote actuated finger exoskeleton using motion-copying system for tendon rehabilitation," IEEE Trans. Industrial Informatics, 2018.

[18] F. Chinello, M. Malvezzi, C. Pacchierotti, and D. Prattichizzo, "A three dofs wearable tactile display for exploration and manipulation of virtual objects," in Proc. IEEE Haptics Symp., 2012, pp. 71-76.

[19] L.-W. Tsai, Robot analysis: the mechanics of serial and parallel manipulators. John Wiley \& Sons, 1999.

[20] L. Sciavicco and B. Siciliano, Modelling and control of robot manipulators. Springer Science \& Business Media, 2000.

[21] G. Gescheider, Psychophysics: the fundamentals. Lawrence Erlbaum, 1997.

[22] H. Stanislaw and N. Todorov, "Calculation of signal detection theory measures," Behavior Research Meth., vol. 31, no. 1, pp. 137-149, 1999.

[23] M. Wijntjes, A. Sato, V. Hayward, and A. Kappers, "Local surface orientation dominates haptic curvature discrimination," IEEE Trans. Haptics, pp. 94-102, 2009.

[24] F. Lee, P. Littrup, S. Torp-Pedersen, C. Mettlin, T. McHugh, J. Gray, G. Kumasaka, and R. McLeary, "Prostate cancer: comparison of transrectal us and digital rectal examination for screening." Radiology, vol 168, no. 2, pp. 389-394, 1988.

[25] J.-H. Ryu, D.-S. Kwon, and B. Hannaford, "Stable teleoperation with time-domain passivity control," IEEE Trans. Robotics and Automation, vol. 20, no. 2, pp. 365-373, 2004.

[26] M. Franken, S. Stramigioli, S. Misra, C. Secchi, and A. Macchelli, "Bilateral telemanipulation with time delays: A two-layer approach combining passivity and transparency," IEEE transactions on robotics, vol. 27, no. 4, pp. 741-756, 2011.

[27] C. Pacchierotti, F. Ongaro, F. Van den Brink, C. Yoon, D. Prattichizzo, D. H. Gracias, and S. Misra, "Steering and control of miniaturized untethered soft magnetic grippers with haptic assistance," IEEE Trans. Automation Science and Engineering, vol. 15, no. 1, pp. 290-306, 2018.

[28] S. Scheggi, L. Meli, C. Pacchierotti, and D. Prattichizzo, "Touch the virtual reality: using the leap motion controller for hand tracking and wearable tactile devices for immersive haptic rendering," in Proc. ACM SIGGRAPH Posters, 2015, p. 31.

[29] L. Meli, S. Scheggi, C. Pacchierotti, and D. Prattichizzo, "Wearable haptics and hand tracking via an rgb-d camera for immersive tactile experiences," in Proc. ACM SIGGRAPH Posters, 2014.

[30] L. Meli, C. Pacchierotti, G. Salvietti, F. Chinello, M. Maisto, A. De Luca, and D. Prattichizzo, "Combining wearable finger haptics and augmented reality: User evaluation using an external camera and the microsoft hololens," IEEE Robotics and Automation Letters, vol. 3 , no. 4, pp. 4297-4304, 2018.

[31] D. C. Ruspini, K. Kolarov, and O. Khatib, "Haptic interaction in virtual environments," in Proc. IEEE/RSJ International Conference on Intelligent Robots and Systems, vol. 1, 1997, pp. 128-133.

[32] A. M. Lund, "Measuring usability with the use questionnaire12," Usability interface, vol. 8, no. 2, pp. 3-6, 2001.

[33] S. De, J. Rosen, A. Dagan, B. Hannaford, P. Swanson, and M. Sinanan, "Assessment of tissue damage due to mechanical stresses," The Int. Journal of Robotics Research, vol. 26, no. 11-12, pp. 1159-1171, 2007.

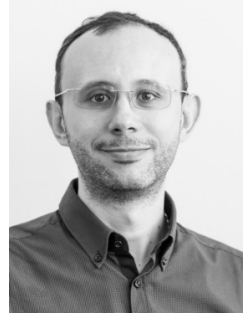

Francesco Chinello (M'19) is an Assistant Professor of the Department of Business Development and Technology at the Aarhus University, Denmark. He earned his Ph.D. degree in Automatic Control and Robotics from the University of Siena in 2014. Chinello was previously a postdoctoral researcher at University of Siena and fellow at the Italian Institute of Technology, from 2014 to 2016. He also visited the Neural Control of Movement Laboratory of the Arizona University in 2013 and 2014 . His research interests include robotics, control, design of haptic interfaces, human-robo interaction, and human-human interaction through wearable devices.

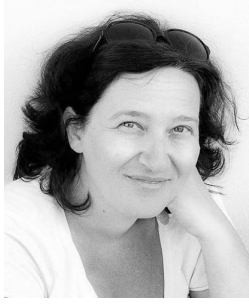

Monica Malvezzi (M'12) is Associate Professor at the University of Siena and Visiting Scientist at the Istituto Italiano di Tecnologia in Genova. She earned her Ph.D. degree in applied mechanics from the University of Bologna in 2003. She has also been Associate Professor of mechanics and mechanism theory at the University of Siena from 2008 to 2018, and Researcher at the University of Florence from 2002 to 2008. Her main research interests include control of mechanical and mechatronic systems, robotics, haptics, vehicle localization, multibody dynamics, grasping, and dexterous manipulation.

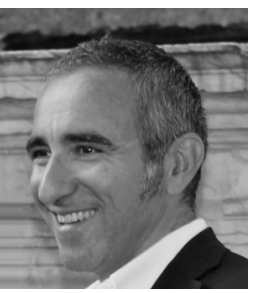

Domenico Prattichizzo (S'93, M'95, F'15) is Full Professor of Robotics at the University of Siena, Senior Scientist at the Istituto Italiano di Tecnologia in Genova, Fellow of the IEEE, and co-founder of WEART, a startup company for VR and AR applications. He earned his Ph.D. in Robotics and Automation at the University of Pisa in 1995. In 1994, he was Visiting Scientist at the MIT AI Lab. Since 2014, Prattichizzo has been an Associate Editor of Frontiers of Robotics and Al. From 2007 to 2013 he was Associate Editor in Chief of the IEEE Transactions on Haptics. From 2003 to 2007, he was an Associate Editor of the IEEE Transactions on Robotics and the IEEE Transactions on Control Systems Technologies. He was also Chair of the Italian Chapter of the IEEE RAS (2006-2010), awarded with the IEEE 2009 Chapter of the Year Award. He is President of the Eurohaptics Society.

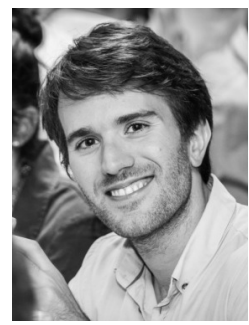

Claudio Pacchierotti (S'12, M'15) is a tenured researcher of the CNRS at IRISA and Inria Rennes Bretagne Atlantique, Rennes, France. He was previously a postdoctoral researcher of the Dept. Advanced Robotics at the Italian Institute of Technology, Genova, Italy. Pacchierotti earned his Ph.D. degree at the University of Siena in 2014. He visited the Penn Haptics Group at the University of Pennsylvania in 2014, the Dept. Innovation in Mechanics and Management of the University of Padua in 2013, and the Institute for Biomedical Technology and Technical Medicine (MIRA) of the University of Twente in 2014. Pacchierotti received the 2014 EuroHaptics Best PhD Thesis Award for the best doctoral thesis in the field of haptics, and the 2015 Meritorious Service Award for his work as a Reviewer for the IEEE Transactions on Haptics. He has also been an Associate Editor for various haptic conferences as well as the Publicity Chair for the 2017 IEEE World Haptics and 2018 Asia Haptics conferences. He is Chair of the IEEE Technical Committee on Haptics and Secretary of the Eurohaptics Society. 